This is the accepted version of the article published by Springer Verlag: lusem, AN., Martínez-Legaz, JE., Todorov, MI., Motzkin predecomposable sets, Journal of Global Optimization (2014) Vol.60 (4), pp. 635-647. The final version is available at: https://doi.org/10.1007/s10898-013-0097-3

\title{
Motzkin predecomposable sets
}

\author{
A.N. Iusem* \\ J.E. Martínez-Legaz ${ }^{\dagger}$ \\ M.I. Todorov ${ }^{\ddagger}$
}

July 23, 2013

\begin{abstract}
We introduce and study the family of sets in a finite dimensional Euclidean space which can be written as the Minkowski sum of a compact and convex set and a convex cone (not necessarily closed). We establish several properties of the class of such sets, called Motzkin predecomposable, some of which hold also for the class of Motzkin decomposable sets (i.e., those for which the convex cone in the decomposition is requested to be closed), while others are specific of the new family.
\end{abstract}

Key words: Motzkin decomposable sets, convex sets, convex cones.

Mathematical Subject Classification (2010): 52A07, 54F65.

\footnotetext{
*Instituto de Matématica Pura e Aplicada (IMPA), Estrada Dona Castorina 110, Rio de Janeiro, RJ, CEP 22460-320, Brazil. The work of this author author was partially supported by CNPq grant no. 301280/86.

†Department of Economics and Economic History, Universitat Autònoma de Barcelona, 08193 Bellaterra, Spain. This author has been supported by the MICINN of Spain, Grant MTM2011-29064-C03-01. He is affiliated to MOVE (Markets, Organizations and Votes in Economics).

${ }^{\ddagger}$ Department of Actuary and Mathematics, Universidad de las Américas, Cholula, Puebla, México. On leave IMI-BAS, Sofia Bulgaria.
} 


\section{Introduction}

It has been known at least since the mid XIX century that the set of solutions of a system of linear equations admits an explicit representation, as the sum of a particular solution plus an arbitrary linear combination of the vectors forming a basis of the linear subspace consisting of the solutions of the associated homogeneous system. The corresponding result for systems of linear inequalities is much more recent. In his doctoral thesis [10], written in 1936, T. Motzkin proved that the set $F$ of solutions of such a system consists of the sums of convex combinations of a finite set of vectors (the vertices of $F$ ) and nonnegative combinations of another finite set (the extreme rays of $F$ ). In modern terminology, every (possibly unbounded) polyhedron in $\mathbb{R}^{n}$ is the Minkowski sum of a convex and compact polyhedron and a closed and convex cone. This characterization turned out to be quite useful for establishing finite convergence of pivotal algorithms for Linear Programming (e.g. the Simplex Method, see [3]), and more specifically, for Quadratic Programming, like Lemke's method, see [1].

Motzkin's representation result suggested the consideration of a class of convex sets more general than polyhedra, resulting from removing the "linear" nature of these, while keeping the decomposition aspect. More precisely, those sets in $\mathbb{R}^{n}$ which can be written as the Minkowski sum of a compact and convex set and a closed and convex cone. Such sets were introduced in [4], where they were called Motzkin decomposable, or M-decomposable, in short, and further studied in [6] and [5], together with the M-decomposable functions, namely those whose epigraphs are M-decomposable. The class of M-decomposable sets lies hence in between the classes of closed polyhedra and of closed and convex sets.

We comment now on some features of M-decomposable sets. It is easy to check that the cone $D$ appearing in the decomposition of an M-decomposable set $F=C+D$ (where $C$ is the compact set), is uniquely determined; it is precisely the recession cone $0^{+} F$ of $F$, namely the set of directions $d \in \mathbb{R}^{n}$ such that $\left\{a+t d: t \in \mathbb{R}_{+}\right\} \subset F$ for some $a \in F$. On the other hand, whenever $D \neq\{0\}$, the compact and convex set $C$ is not uniquely determined; the given set $C$ might be replaced, for instance, by $F \cap B$, where $B$ is any ball in $\mathbb{R}^{n}$ containing $C$.

M-decomposable sets for which the cone $D$ is pointed (i.e., it contains no lines), have a clear geometric characterization: they are precisely those closed and convex sets with a bounded set of extreme points [6, Theorem 11.(i)].

A sizable number of additional properties of such sets and functions were established in the above mentioned references, and we single out two of them for future reference: first, faces of M-decomposable sets are themselves Mdecomposable [5, Corollary 3], and second, M-decomposable sets whose recession cones are pointed, admit a minimal decomposition, which we define next. We say that a representation $F=C+D$ of an M-decomposable set $F$ is minimal when $C \subset C^{\prime}$ for any convex and compact set $C^{\prime} \subset \mathbb{R}^{n}$ such that $F=C^{\prime}+D$. When $F$ is M-decomposable and $D$ is pointed, there exists such a minimal decomposition, and the corresponding compact and convex set $C$ is itself uniquely determined: it is the closed and convex hull of the extreme points of $F[6$, 
Theorem 11.(ii)].

In connection with this property, we mention, parenthetically, that it does not hold when $D$ is not pointed: if $F$ is a line, then any singleton in $F$ can be taken as $C$, but there exist minimal decompositions $F=D+C$ in a weaker sense, meaning that there exists no closed and convex set $C^{\prime}$ such that $F=C^{\prime}+D$ and $C^{\prime}$ is strictly contained in $C$. In fact, when $D$ is not pointed, we can "separate" the linear part of $D$ in the following way. Let $L$ be the linearity of $D$, i.e. the set of directions of lines contained in $D$. $L$ is a linear subspace, and we can write $F=C+D$ as $F=C+\left[L+\left(D \cap L^{\perp}\right)\right]=L+\left(C+D^{\prime}\right)$, where $L^{\perp}$ is the orthogonal complement of $L$ and $D^{\prime \perp}$. Now $D^{\prime}$ is pointed and the M-decomposable set $C+D^{\prime}$ has a minimal decomposition of the form $\bar{C}+D^{\prime}$ in the strong sense, and it follows easily that $\bar{C}+\left(L+D^{\prime}\right)=\bar{C}+D$ is a minimal decomposition of $F$ in the weak sense. The fact that $\bar{C}$ is not unique is made clear if we observe that we can replace $L^{\perp}$ by $a+L^{\perp}$ in the above described separation procedure, with an arbitrary $a \in L$, in which case we end up with $a+\bar{C}$ instead of $\bar{C}$. If we adopt the convention that the separation procedure must be performed with a linear subspace (i.e., not with an affine manifold away from 0), then we recover the minimal decomposition property in the strong sense: the minimal compact component of $F$ is taken as the minimal component of $F \cap L^{\perp}$. It follows from this discussion that no generality is lost if we restrict out attention to M-decomposable sets whose recession cones are pointed.

As we have commented, the class of M-decomposable sets shares many properties of the class of convex sets, but several important ones are not inherited by the smaller class. We focus on three of them. It is well known that both the Minkowski sum and the intersection of two convex sets are convex, as well as the image of a convex set through an affine map. These three properties fail for M-decomposable sets, as we describe next.

For the intersection, the ancient Greek geometers already knew that the intersection of a "classical" cone with a plane parallel to its axis is a hyperbola. In modern notation, let $F_{1}$ be the "ice cream" cone in $\mathbb{R}^{3}$, i.e. $F_{1}=\{(r, s, t)$ : $\left.t \geq 0, t^{2} \geq r^{2}+s^{2}\right\}$, and $F_{2}$ the plane normal to $e^{1}=(1,0,0)$ passing through $e^{1}$, i.e., $F_{2}=\{(1, s, t): s, t \in \mathbb{R}\}$. Both $F_{1}$ and $F_{2}$ are M-decomposable $\left(F_{1}\right.$ is a closed and convex cone, and $F_{2}$ is a polyhedron), but

$$
F:=F_{1} \cap F_{2}=\left\{(1, s, t): t \geq 0, t^{2} \geq 1+s^{2}\right\}
$$

is a hyperbola $H$ contained in the plane $F_{2}$. Since all points in $H$ are extreme points of $F$, and $H$ is unbounded, the above mentioned characterization in terms of boundedness of the set of extreme points entails that $F$ is not Mdecomposable.

For the other two properties, let $\mathbb{R}_{++}^{n}$ denote the interior of the nonnegative orthant of $\mathbb{R}^{n}$, and consider the cone $C \subset \mathbb{R}^{3}$ spanned by the closed and convex set $U:=\{(1, s, t):, s>0, s t \geq 1\}$, i.e.,

$$
C=\{(r, r s, r t,): r \geq 0, s>0, s t \geq 1\} .
$$


Let $C^{\prime}$ be the closed halfline through $(0,0,1)$. It is easy to check that $C+$ $C^{\prime}=\mathbb{R}_{++}^{3} \cup\{0\}$. Both $C$ and $C^{\prime}$ are closed and convex cones, hence Mdecomposable, while $C+C^{\prime}$ is a convex cone, but is not closed, and hence is not M-decomposable. Take now the orthogonal projection $P: \mathbb{R}^{3} \rightarrow \mathbb{R}^{2}$ given by $P(r, s, t)=(r, s)$. It is easy to check that $P(C)=\mathbb{R}_{++}^{2} \cup\{0\} . C$ is Mdecomposable, $P$ is linear, but $P(C)$ is a nonclosed convex cone, and therefore it is not M-decomposable.

It seems that there is not much to be done in connection with the intersection property, in particular if one tries to identify a reasonable subclass of the family of M-decomposable sets which is closed through intersections (besides, of course, the trivial one, namely when both decomposable sets are themselves polyhedra, in which case the intersection is also a polyhedron). If we look at two Mdecomposable sets $F_{i}=C_{i}+D_{i}(1 \leq i \leq 2)$ and search for properties on the $C_{i}$ 's, $D_{i}$ 's which ensure that $F_{1} \cap F_{2}$ is M-decomposable, we observe that in the counterexample above both $C_{i}$ 's can be taken as singletons (hence polyhedra), one of the cones is also a polyhedron (the one corresponding to $F_{2}$ ) and only $F_{1}$ is non-polyhedral (and it has only one extreme point). There are also examples in which $D_{1}$ and $D_{2}$ are polyhedral cones and $C_{1}$ is a singleton, but nevertheless $F_{1} \cap F_{2}$ is not M-decomposable.

On the other hand, if we consider Minkowski sums and images through affine mappings, we immediately see that the problem is not related to the decomposability or the convexity, but just to the closedness. In fact, such properties are enjoyed by the class of convex sets, but not by the class of closed and convex sets. It is thus natural to attempt to improve the regularity properties of the class of M-decomposable sets by enlarging, rather than decreasing it; more specifically, by removing the closedness requirement. We emphasize that closedness appears twice in the definiton of an M-decomposable set: both $C$ and $D$ are required to be closed. However, the closedness of $C$ seems harmless enough: the Minkowski sum of two compact sets in compact, hence closed, and compactness, being a topological invariant, is preserved not only by affine mappings but by arbitrary continuous ones. Indeed, the candidate for removal is the closedness requirement on the convex cones.

We consider thus subsets of $\mathbb{R}^{n}$ which are sums of a compact and convex set and a convex cone, not necessarily closed. Such sets will be called in the sequel Motzkin predecomposable, or M-predecomposable, for short. We will show that the class of M-predecomposable sets is indeed invariant by afine mappings and Minkoswki sums (see Proposition 13 in Section 2). At the same time, it is important to assess the possible ill consequences of such an enlargement; it would not be worthwhile to consider the new class if by removing the closedness assumption on the cone we lose the already established good properties of the class of M-decomposable sets. Fortunately, this is not the case, and the purpose of this paper is to establish this fact. In particular, we will prove that the faces of M-predecomposable sets are M-predecomposable (Proposition 14 in Section 2), as well as the existence of minimal decompositions of M-predecomposable sets whose asssociated cones are pointed (Theorem 29 in Section 2). Additionally we will provide two characterizations of M-predecomposable sets, both related 
to some properties of their faces (Theorems 15 and 25 in Section 2).

We observe that the elimination of the closedness assumption on the cones causes produces many technical complications, and thus the results on Mpredecomposable sets require proofs quite different from their counterparts for M-decomposable ones. We also remark that this paper contains a very preliminary study of M-predecomposable sets. In a certain sense, our goal has been just to exhibit some evidence showing that this new class deserves indeed consideration. A more complete study of M-predecomposable sets, recovering as many as possible of the results known to hold for M-decomposable sets, is left for future research.

\section{Motzkin predecomposable sets}

We start this section with the formal definition of M-predecomposable sets.

Definition 1. A nonempty set $F \subset \mathbb{R}^{n}$ is Motzkin predecomposable (M-predecomposable in short) if there exists a compact convex set $C$ and a convex cone $D$ such that $F=C+D$. The pair $(C, D)$ will be called a Motzkin decomposition of $F$, and the set $C$ will be called a compact component of $F$.

Next, we present several elementary properties of M-predecomposable sets, as well as the precise relation between the notions of M-decomposability and M-predecomposability.

Remark 2. It is clear that every cone $D \subset \mathbb{R}$ is either $\{0\}$ or a closed halfine. In both cases $D$ is "a fortiori" closed, so that every M-predecomposable set $F \subset \mathbb{R}$ is $M$-decomposable. In fact, it is easy to see that a nonempty set $F \subset \mathbb{R}$ is M-predecomposable if and only if it is convex and closed.

Every M-predecomposable set is convex, because it is the sum of two convex sets.

Proposition 3. A nonempty set $F \subset \mathbb{R}^{n}$ is $M$-predecomposable if and only if it is convex and there exist a compact set $C$ and a cone $D$ such that $F=C+D$. In this case, (convC, convD) is a Motzkin decomposition of $F$.

Proof. We only need to prove the "if" statement. It follows from the equalities $F=\operatorname{conv} F=\operatorname{conv}(C+D)=\operatorname{conv} C+\operatorname{conv} D$ and the fact that the convex hull of a compact set (of a cone) is compact (a cone, respectively).

We will make an extensive use of the following lexicographical separation theorem:

Theorem 4 (Lexicographical Separation Theorem). ([7, p. 258], [9, Theorem 1.1]) Let $C$ be a convex subset of $\mathbb{R}^{n}$ and $x_{0} \in \mathbb{R}^{n} \backslash C$. Then there exists an $n \times n$ matrix $M$ such that $M x<_{L} M x_{0}$ for all $x \in C$.

The following result is well known under the extra assumption that the set $B$ is closed (see [11, p. 493]). 
Proposition 5 (Order Cancellation Law). Let $A, B, C \subset \mathbb{R}^{n}$. If $A$ and $B$ are convex, $C$ is nonempty and compact and $A+C \subset B+C$, then $A \subset B$.

Proof. Take $x_{0} \in A$. If $x_{0} \notin B$ then, by Theorem 4 , there exists an $n \times n$ matrix $M$ such that $M x<_{L} M x_{0}$ for all $x \in B$. Take a lexicographical global maximum $z$ over $C$ of the linear mapping $x \longmapsto M x$, that is, $z \in C$ and $M y \leq_{L} M z$ for all $y \in C$. The existence of such a lexicographical maximum follows by successively applying Weierstrass Theorem $n$ times. We thus have $M(x+y)<_{L} M\left(x_{0}+z\right)$ for all $x \in B$ and $y \in C$. Hence $x_{0}+z \notin B+C$, which contradicts the fact that $x_{0}+z \in A+C \subset B+C$. Thus $x_{0} \in B$.

Proposition 6. If $(C, D)$ is a Motzkin decomposition of a nonempty set $F \subset$ $\mathbb{R}^{n}$, then $D=0^{+} F$.

Proof. Clearly, $D \subset 0^{+} F$. On the other hand, $C+0^{+} F \subset F+0^{+} F=F=C+D$; hence, by Proposition $5,0^{+} F \subset D$. We thus have $D=0^{+} F$.

For $a \in \mathbb{R}^{n}$ and $G \subset \mathbb{R}^{n}$, the set of minimizers of the linear function $x \longmapsto a^{\prime} x$ over $G$ will be denoted by $G^{*}(a)$.

Remark 7. Clearly, every $M$-decomposable set is $M$-predecomposable, too. In view of Proposition 6, for $M$-decomposable sets the $M$-decompositions in the sense of such sets coincide with the M-decompositions in the sense of Definition 1. In particular, the compact components also coincide.

Proposition 8. If $F \subset \mathbb{R}^{n}$ is $M$-predecomposable, then clF is $M$-decomposable and $0^{+} \mathrm{clF}=\mathrm{cl} 0^{+} \mathrm{F}$.

Proof. It follows from the equalities $c l F=c l\left(C+0^{+} F\right)=C+c l 0^{+} F$, using Proposition 6.

Corollary 9. A closed set $F \subset \mathbb{R}^{n}$ is $M$-predecomposable if and only if it is M-decomposable.

We will denote by econvF the evenly convex hull of a set $F \subset \mathbb{R}^{n}$. Let us recall that a set is evenly convex when it is the intersection of open halfspaces (or equivalently, of open convex sets, see e.g. [2]). Since every closed convex set is evenly convex and every evenly convex set is convex, one has conv $F \subset$ econvF $\subset$ cl convF.

Proposition 10. If $F \subset \mathbb{R}^{n}$ is $M$-predecomposable, then econvF $=c l F$.

Proof. Let $x \in \mathbb{R}^{n} \backslash e \operatorname{conv} F$ and $C$ be a compact component of $F$. Then there exists $a \in \mathbb{R}^{n}$ such that

$$
a^{\prime}(c+d)<a^{\prime} x \text { for every } c \in C \text { and } d \in 0^{+} F \text {. }
$$

Take $\bar{c} \in C$ such that $a^{\prime} c \leq a^{\prime} \bar{c}$ for every $c \in C$. Setting $c=\bar{c}$ and $d=0$ in (1), we get $a^{\prime} \bar{c}<a^{\prime} x$. Hence, by (1) with $c=\bar{c}$, we have $a^{\prime} d<a^{\prime} x-a^{\prime} \bar{c}$ for every $d \in 0^{+} F$. Since $0^{+} F$ is a cone, it follows that $a^{\prime} d \leq 0$ for every $d \in 0^{+} F$. Therefore, for every $c \in C$ and $d \in 0^{+} F$ we have $a^{\prime}(c+\bar{d}) \leq a^{\prime} \bar{c}<a^{\prime} x$, which, since $F=C+0^{+} F$, shows that $x \notin c l F$. 
Corollary 11. If $F \subset \mathbb{R}^{n}$ is $M$-predecomposable and evenly convex, then it is closed.

Proof. We have $F=e c o n v F=c l F$.

Corollary 12. If $F \subset \mathbb{R}^{n}$ is $M$-predecomposable and open, then $F=\mathbb{R}^{n}$.

Proof. Since every open convex set is evenly convex, from Corollary 11 it follows that $F$ is closed. It only remains to recall that $F \neq \emptyset$ and $\mathbb{R}^{n}$ is connected.

We continue with the two properties of the class of M-predecomposable sets which are not valid for the class of M-decomposable sets, discussed in Section 1.

Proposition 13. (i) If $F_{1}$ and $F_{2}$ are $M$-predecomposable then $F=F_{1}+F_{2}$ is M-predecomposable.

(ii) If $F$ is $M$-predecomposable and $E: \mathbb{R}^{n} \rightarrow \mathbb{R}^{m}$ is affine, then $E(F) \subset \mathbb{R}^{m}$ is M-predecomposable.

Proof. (i) Let $\left(C_{1}, D_{1}\right),\left(C_{2}, D_{2}\right)$ be decompositions of $F_{1}, F_{2}$ respectively. Then $F=C+D$ with $C=C_{1}+C_{2}, D=D_{1}+D_{2}$, and it suffices to invoke the well known facts that the sum of two convex sets (two compact sets, two cones, respectively) is a convex set (a compact set, a cone, respectively).

(ii) Since $E$ is affine, there exist $A \in \mathbb{R}^{m \times n}, b \in \mathbb{R}^{m}$ such that $E(x)=A x+b$. It is easy to check that if $(C, D)$ is a decomposition of $F$ then $E(F)=$ $[b+A(C)]+A(D)$. By linearity of the mapping $x \mapsto A x$, we get that $A(D)$ is a cone and that $A(C)$ is convex and compact, so that the same holds for $b+A(C)$. Thus $(b+A(C), A(D))$ is a decomposition of $E(F)$, which is therefore M-predecomposable.

Next, we prove one of the properties commented upon in Section 1, namely that faces of M-predecomposable sets are themselves M-predecomposable.

Proposition 14. Every nonempty face of an $M$-predecomposable face is $M$ predecomposable, too. More specifically, if $C$ is a compact component of $F$ and $G$ is a nonempty face of $F$, then $C \cap G=C \cap$ aff $G$ is a compact component of $G$.

Proof. Let $G$ be a nonempty face of an M-predecomposable set $F$ and $C$ be a compact component of $F$. We will prove that $G=C \cap G+0^{+} G$. Since $C \cap G \subset C \cap$ aff $G \subset F \cap$ aff $f=G$, the inclusion $\supset$ is obvious; moreover, by taking the intersection with $G$ it follows that $C \cap G=C \cap$ aff $G$, which shows that $C \cap G$ is compact. For proving the inclusion $G \subset C \cap G+0^{+} G$, let $x \in G$. Since $G \subset F=C+D=C+0^{+} F$, there exist $c \in C$ and $d \in 0^{+} F$ such that $x=c+d$. For every $\lambda>1$ we have $x=\left(1-\frac{1}{\lambda}\right) c+\frac{1}{\lambda}(c+\lambda d)$; hence, given that $c, c+\lambda d \in F$, we conclude that $c \in G$ and $c+\lambda d \in G$, from which we 
deduce that $d \in 0^{+} G$. Since $c \in C \cap G$, it follows that $x \in C \cap G+0^{+} G$. We have thus proved that $C \cap G$ is a compact component of $G$.

The next result characterizes the M-predecomposability of a convex set in terms of certain properties of its faces.

Theorem 15. Let $F \subset \mathbb{R}^{n}$ be a nonempty convex set. Then the following statements are equivalent:

(a) $F$ is M-predecomposable.

(b) The following conditions hold:

1) $c l 0^{+} \mathrm{F}=0^{+} \mathrm{clF}$.

2) For every nonempty face $G$ of $F$, one has

$$
0^{+} G \subset 0^{+} F \text {. }
$$

3) There exists a compact convex set $C \subset F$ such that

(i) $F^{*}(a) \cap C \neq \emptyset$ for each $a \in \mathbb{R}^{n}$ such that the linear function $x \longmapsto a^{\prime} x$ is bounded from below on $F$.

(ii) for every exposed face $G$ of $F$, the set $C \cap G$ is a compact component of $G$.

(c) The same as b), with 2) stated only for every exposed face $G$ of $F$ and (ii) replaced by the following condition:

(ii') for every supporting hyperplane $H$ of $F$ such that $\left(c l 0^{+} F \backslash 0^{+} F\right) \cap 0^{+} H \neq$ $\emptyset$, the set $C \cap H$ is a compact component of $F \cap H$.

In such a case, a compact convex set $C \subset \mathbb{R}^{n}$ is a compact component of $F$ if and only if it satisfies the properties stated in (i) and (ii) or, equivalently, in (i) and (ii').

Proof. (a) $\Longrightarrow$ (b). By Proposition 8, property 1 ) holds. Let $C$ be a compact component of $F$. For proving 2), let $c \in C \cap$ aff $G$ and $d \in 0^{+} G$. By Proposition 14, we have $c+d \in C \cap$ aff $G+0^{+} G=G \subset F=C+0^{+} F$; hence there exist $c^{\prime} \in C$ and $d^{\prime} \in 0^{+} F$ such that $c+d=c^{\prime}+d^{\prime}$. Since $c^{\prime}+2 d^{\prime} \in F$ and $G$ is a face of $F$, from the equality $c^{\prime}+d^{\prime}=\frac{1}{2}\left(c^{\prime}+\left(c^{\prime}+2 d^{\prime}\right)\right)$ we deduce that $c^{\prime} \in G \subset$ aff $G$; therefore $c^{\prime} \in C \cap a f f G$. We have shown that $C \cap a f f G+0^{+} G \subset C \cap a f f G+0^{+} F$, which, using Proposition 5, yields (2). For proving 3), notice that property (i) clearly holds and, by Proposition 14, property (ii) holds too.

Implication (b) $\Longrightarrow$ (c) is obvious, since $F \cap H$ is an exposed face of $F$.

(c) $\Longrightarrow$ (a). Let $a \in \mathbb{R}^{n}$ be such that the linear function $x \longmapsto a^{\prime} x$ is bounded from below on $c l F$. Then this linear function is bounded from below on $F$ as well and hence, by (i), we have $F^{*}(a) \cap C \neq \emptyset$. Since $F^{*}(a) \subseteq(c l F)^{*}(a)$, it follows that $(c l F)^{*}(a) \cap C \neq \emptyset$. Therefore, in view of the proof of $[4$, Proposition 16], the set $c l F$ is M-decomposable and $C$ is a compact component of $F$. Thus, 
using 1) we get $C+0^{+} F \subset F \subset c l F=C+0^{+} c l F=C+c l 0^{+} F=c l\left(C+0^{+} F\right)$. Suppose that $C+0^{+} F \neq F$, and take a point $x \in F \backslash\left(C+0^{+} F\right)$. Then $x \in F \backslash \operatorname{int}\left(C+0^{+} F\right)=F \backslash$ int $c l\left(C+0^{+} F\right)=F \backslash$ int $c l F=F \backslash$ int $F \subset$ $b d F$. We can thus take a supporting hyperplane $H$ of $F$ at $x$. Since $x \in$ $c l F=C+c l 0^{+} F$, there exist $c \in C$ and $d \in c l 0^{+} F$ such that $x=c+d$. As $x \notin C+0^{+} F$, we have $d \notin 0^{+} F$, so that $d \in\left(c l 0^{+} F \backslash 0^{+} F\right)$. On the other hand, $H$ is a supporting hyperplane of $c l F$ at $x$ too, and therefore $(c l F) \cap H$ is an exposed face of $c l F$. Hence, given that $x \in(c l F) \cap H$ and $c, c+2 d \in c l F$, from the equalities $x=c+d=\frac{1}{2}(c+(c+2 d))$ we deduce that $c, c+2 d \in(c l F) \cap H$, which implies that $d \in 0^{+} H$. We thus have $d \in\left(c l 0^{+} F \backslash 0^{+} F\right) \cap 0^{+} H$ and therefore, by (ii'), the set $C \cap H$ is a compact component of $F \cap H$. Hence, by (2), we have $x \in F \cap H=C \cap H+0^{+}(F \cap H) \subset C+0^{+} F$. This is a contradiction, so we must have $C+0^{+} F=F$. Thus, $F$ is M-predecomposable.

In view of the proof of the implication $(\mathrm{c}) \Longrightarrow(\mathrm{a})$, if a compact convex set $C \subset \mathbb{R}^{n}$ satisfies properties (ii) and (ii'), then $F=C+0^{+} F$, that is, $C$ is a compact component of $F$. Conversely, if $C$ is a compact component of $F$ then, by Proposition 14, property (ii) holds too.

The theorem is proved.

We continue with four examples, showing that none among conditions 1), 2), 3)(i) and 3)(ii) in the statement of Theorem 15 is superfluous.

Example 16. The convex set $F:=(] 0,1[\times[0,+\infty[) \cup(\{0,1\} \times[0,1])$ satisfies conditions 2) and 3) of Theorem 15, with $C:=[0,1] \times[0,1]$, but not condition 1), because $0^{+} \mathrm{cl} F=\{0\} \times\left[0,+\infty\left[\right.\right.$ and $0^{+} F=\{0\}$. Thus $F$ is not $M$ predecomposable. This shows that condition 1) is not superfluous in the statement of Theorem 15.

Example 17. The convex set

$$
F:=([0,+\infty[\times[0,+\infty[\times[0,1]) \backslash(] 0,+\infty[\times\{0\} \times\{0\})
$$

satisfies conditions 1) and 3) of Theorem 15, with $C:=\{0\} \times[0,1] \times[0,1]$, but not condition 2$)$, because for the face $G:=] 0,+\infty[\times\{0\} \times\{1\}$ one has $0^{+} G=[0,+\infty[\times\{0\} \times\{0\}$, whereas

$$
0^{+} F=([0,+\infty[\times[0,+\infty[\times\{0\}) \backslash(] 0,+\infty[\times\{0\} \times\{0\}) .
$$

Thus $F$ is not M-predecomposable. This shows that condition 2) is not superfluous in the statement of Theorem 15.

Example 18. The convex set

$$
F:=\left\{(x, y) \in \mathbb{R}^{2}: x>0, y>0, x y>1\right\} \cup\{(1,1)\}
$$

satisfies conditions 1) and 2) and 3)(ii) of Theorem 15, with $C:=\{(1,1)\}$ (which is actually the only proper face of $F$ ), but there is no compact convex set $C$ satisfying condition 3 ) ( $i$ ) (indeed, consider, for instance, the vector $a:=$ $(1,0))$. Thus $F$ is not M-predecomposable. This shows that condition 3$)(i)$ is not superfluous in the statement of Theorem 15. 
Example 19. The convex set

$$
F:=([0,1] \times[0,+\infty[\times[0,+\infty[) \backslash(\{0,1\} \times\{0\} \times[0,+\infty[)
$$

satisfies conditions 1$)$ and 2 ) and 3$)(i)$ of Theorem 15 , with $C:=[0,1] \times\{0\} \times$ $\{0\}$, but since the exposed face

$$
G:=([0,1] \times\{0\} \times[0,+\infty[) \backslash(\{0,1\} \times\{0\} \times] 0,+\infty[)
$$

is not M-predecomposable (hence, by Proposition 14, $F$ is not M-predecomposable either), no compact set $C$ satisfies condition 3$)($ ii). Thus $F$ is not $M$-predecomposable. This shows that condition 3)(ii) is not superfluous in the statement of Theorem 15.

We recover now a property of M-decomposable sets, established in [4].

Corollary 20. [4, Proposition 16] A closed convex set $F \subset \mathbb{R}^{n}$ is $M$-decomposable if and only if there exists a compact set $C \subset F$ such that $F^{*}(a) \cap C \neq \emptyset$ for each $a \in \mathbb{R}^{n}$ such that the linear function $x \longmapsto a^{\prime} x$ is bounded from below on $F$.

Proof. Combine Corollary 9 with equivalence (a) $\Longleftrightarrow$ (c) in Theorem 15, taking into account that for a closed convex set $F$ properties 1), 2) and (ii') hold (the latter, vacuously).

The following characterization of faces of convex sets will be useful to obtain another characterization of M-predecomposable sets:

Theorem 21. [8, Thm. 2] Let $F \subset \mathbb{R}^{n}$ be a convex set and $\emptyset \neq G \subset F$. Then $G$ is a face of $F$ if and only if there exists a $k \times n$ matrix A, for some $k \in\{1, \ldots, n\}$, such that

$$
G=\left\{y \in F: A y=\min _{L}\{A x: x \in F\}\right\} .
$$

Lemma 22. Let $F \subset \mathbb{R}^{n}$ be a nonempty convex set such that $0^{+} c l F=c l 0^{+} F$ and $C$ be a compact subset of $F$ satisfying $F^{*}(a) \cap C \neq \emptyset$ for each $a \in \mathbb{R}^{n}$ such that the linear function $x \longmapsto a^{\prime} x$ is bounded from below on $F$. If $a \in \mathbb{R}^{n}$ satisfies $a^{\prime} d \geq 0$ for every $d \in 0^{+} F$, then $F^{*}(a) \cap C \neq \emptyset$.

Proof. Since $a$ belongs to the positive polar cone of $c l 0^{+} F=0^{+} c l F$, that polar cone coincides with the closure of the barrier set $\mathbb{B}(c l F)$, defined as

$$
\left\{b \in \mathbb{R}^{n} \text { : the linear function } x \longmapsto b^{\prime} x \text { is bounded from below on } c l G\right\} .
$$

We thus have $a=\lim b_{k}$ for some sequence $b_{k}$ in $\mathbb{B}(c l F)$. By assumption, for each $k$ one has $F^{*}\left(b_{k}\right) \cap C \neq \emptyset$. Take $c_{k} \in F^{*}\left(b_{k}\right) \cap C$. For every $x \in F$, we have $b_{k}^{\prime} x \geq b_{k}^{\prime} c_{k}$. In view of the compactness of $C$, we can assume, without loss of generality, that the sequence $c_{k}$ converges to some $c \in C$. Taking limits in the inequality $b_{k}^{\prime} x \geq b_{k}^{\prime} c_{k}$, we get $a^{\prime} x \geq a^{\prime} c$, which shows that $F^{*}(a) \cap C \neq \emptyset$. 
We present now another auxiliary result, dealing with existence of lexicographical minimizers of affine functions on convex sets.

Lemma 23. Let $F \subset \mathbb{R}^{n}$ be a nonempty convex set satisfying the following two properties:

(i) There exists a compact set $C \subset F$ such that, for every nonempty face $G$ of $F$, it holds that $G^{*}(a) \cap C \neq \emptyset$ for each $a \in \mathbb{R}^{n}$ such that the linear function $x \longmapsto a^{\prime} x$ is bounded from below on $G$.

(ii) For every nonempty face $G$ of $F$, it holds that $0^{+} \mathrm{cl} G=c l 0^{+} G$.

If $A$ is a $k \times n$ matrix such that $A d \geq_{L} 0$ for every $d \in 0^{+} F$, then the linear mapping $x \longmapsto A x$ attains a lexicographical minimum over $F$ at some point $c \in C$.

Proof. We will proceed by induction on $k$. For $k=1$, the statement is an immediate consequence of Lemma 22. Take $k>1$ and denote by $A_{k-1}$ the matrix obtained by deleting the last row $a_{k}$ of $A$. Clearly, $A_{k-1} d \geq_{L} 0$ for every $d \in 0^{+} F$; hence, by the induction hypothesis, the linear mapping $x \longmapsto A_{k-1} x$ attains a lexicographical minimum over $F$ at some point $\bar{c} \in C$. By Theorem 21 , the set $G:=\left\{x \in F: A_{k-1} x=A_{k-1} \bar{c}\right\}$ is a (nonempty) face of $F$. Take $d \in 0^{+} G$. Clearly, $d \in 0^{+} F$; hence, $A d \geq_{L} 0$. Since we also have $A_{k-1} d=0$, it follows that $a_{k}^{\prime} d \geq 0$. Therefore, by Lemma 22, we have $G^{*}\left(a_{k}\right) \cap C \neq \emptyset$. Take $c \in G^{*}\left(a_{k}\right) \cap C$. For every $x \in F$ we have $A_{k-1} x \geq_{L} A_{k-1} \bar{c}=A_{k-1} c$ and, if $A_{k-1} x=A_{k-1} c$, then $A_{k-1} x=A_{k-1} \bar{c}$, that is, $x \in G$, which implies that $a_{k}^{\prime} x \geq a_{k}^{\prime} c$. We have shown that $A x \geq_{L} A c$ for every $x \in F$.

The next result simplifies the task of checking the assumptions of Lemma 23.

Lemma 24. For a compact set $C \subset F$ to be as in (i) of Lemma 23 it is sufficient that it satisfy this condition for every nonempty face $G$ of $F$ which is either nonclosed or a maximal closed face.

Proof. We only need to prove that (i) holds for an arbitrary nonempty closed face $G$. Using [12, Corollary 18.1.3], it is easy to prove that $G$ is contained in some maximal closed face $\widetilde{G}$. By assumption, the linear mapping $x \longmapsto A x$ attains a lexicographical minimum over $\widetilde{G}$ at some point $c \in C$. On the other hand, by Proposition 14 and Corollary 9 , the face $\widetilde{G}$ is M-decomposable and, in view of the proof of [4, Proposition 16], the set convC $\cap$ aff $\widetilde{G}$ is a compact component of $\widetilde{G}$. Since $G$ is a face of $\widetilde{G}$ (see [12, p. 163]), by Proposition 14 the set convC $\cap a f f G=(\operatorname{conv} C \cap a f f \widetilde{G}) \cap a f f G$ is a compact component of $G$. Hence, by [4, Proposition 13.(v)], if $a \in \mathbb{R}^{n}$ is such that the linear function $x \longmapsto a^{\prime} x$ is bounded from below on $G$ then $G^{*}(a) \cap \operatorname{conv} C \cap$ aff $G \neq \emptyset$. Therefore, $G^{*}(a) \cap \operatorname{conv} C \neq \emptyset$. Since $G^{*}(a)$ is a face of $G$, and hence of $F$ (see [12, p. 163]), it follows that $G^{*}(a) \cap C \neq \emptyset$.

We use now the two previous lemmas in order to provide another characterization of M-predecomposable sets, also related to some properties of their faces. 
Theorem 25. Let $F \subset \mathbb{R}^{n}$ be a nonempty convex set. Then the following statements are equivalent:

(a) $F$ is M-predecomposable.

(b) Conditions (i) and (ii) of Lemma 23 hold.

(c) Conditions (i) and (ii) of Lemma 23 hold for every nonempty face $G$ of $F$ which is either nonclosed or a maximal closed face.

In such a case, a compact set $C \subset \mathbb{R}^{n}$ is such that convC is a compact component of $F$ if and only if it satisfies the property stated in (i).

Proof. Implication (a) $\Longrightarrow$ (b) follows from implication (a) $\Longrightarrow$ (b) of Theorem 15 and Proposition 14.

(b) $\Longrightarrow$ (a). We will prove that $F=C+0^{+} F$. The inclusion $\supset$ is obvious, since $C \subset F$. For proving the opposite inclusion, take $x \in F$ and assume that $x \notin C+0^{+} F$. Then, by Theorem 4 , there exists an $n \times n$ matrix $M$ such that $M x<_{L} M c+M d$ for all $c \in C$ and $d \in 0^{+} F$. For $i=1, \ldots, n$, denote by $M_{i}$ the $i \times n$ submatrix of $M$ obtained by deleting its last $n-i$ rows, and set $m:=\min \left\{i \in\{1, \ldots, n\}: M_{i} x<_{L} M_{i} c+M_{i} d\right.$ for all $c \in C$ and $\left.d \in 0^{+} F\right\}$. We have that

$$
M_{m} x<{ }_{L} M_{m} c+M_{m} d \text { for all } c \in C \text { and } d \in 0^{+} F
$$

and $M_{m-1} x=M_{m-1} \bar{c}+M_{m-1} \bar{d}$ for some $\bar{c} \in C$ and $\bar{d} \in 0^{+} F$, with the convention that $M_{0}$ is the row vector 0 . Since $M_{m-1} x \leq_{L} M_{m-1} c+M_{m-1} d$ for all $c \in C$ and $d \in 0^{+} F$, we have that $M_{m-1} \bar{d}=M_{m-1} x-M_{m-1} \bar{c} \leq_{L} 0$ and $M_{m-1} \bar{d}=$ $M_{m-1} \bar{c}+2 M_{m-1} \bar{d}-\left(M_{m-1} \bar{c}+M_{m-1} \bar{d}\right)=M_{m-1} \bar{c}+2 M_{m-1} \bar{d}-M_{m-1} x \geq_{L} 0 ;$ hence $M_{m-1} \bar{d}=0$ and, consequently, $M_{m-1} x=M_{m-1} \bar{c}$. Therefore, by (3), for every $d \in 0^{+} F$ it holds that $M_{m-1} d \geq_{L} M_{m} x-M_{m} \bar{c}=0$. Hence, by Lemma 23, the linear mapping $y \longmapsto M_{m-1} y$ attains a lexicographical minimum over $F$ at some point $\widehat{c} \in C$. In particular, since $\bar{c} \in F$, we have $M_{m-1} \bar{c} \geq_{L} M_{m-1} \widehat{c}$, but, on the other hand, by (3) we also have $M_{m-1} \widehat{c} \geq_{L} M_{m-1} x=M_{m-1} \bar{c}$, so that $M_{m-1} \widehat{c}=M_{m-1} \bar{c}$. We have shown that $y \longmapsto M_{m-1} y$ attains a lexicographical minimum over $F$ precisely at $\bar{c}$. By Theorem 21, the set $G:=$ $\left\{y \in F: M_{m-1} y=M_{m-1} \bar{c}\right\}$ is a (nonempty) face of $F$. For every $d \in 0^{+} G$ we have $M_{m-1} d=0$; hence, denoting by $r_{m}$ the $m$-th row of $M$, for every $\lambda>0$ we have $M_{m-1} x=M_{m-1} \bar{c}+\lambda M_{m-1} d$. Therefore, by (3), we obtain $r_{m} x<r_{m} \bar{c}+\lambda r_{m} d$, which implies that $r_{m} d \geq 0$. Notice that the faces excluded from statemnent (c) are closed, therefore their recession cones are closed. It thus follows that condition (ii) holds for every nonempty face. Hence, by Lemma 22, $G^{*}\left(r_{m}^{\prime}\right) \cap C \neq \emptyset$. Take $g \in G^{*}\left(r_{m}^{\prime}\right) \cap C$. Since $g \in G$, we have $M_{m-1} g=M_{m-1} \bar{c}=M_{m-1} x$; moreover, taking into account that $x \in G$ and $g \in G^{*}\left(r_{m}^{\prime}\right)$, we also have $r_{m} x \geq r_{m} g$. It thus follows that $M_{m} x \geq_{L} M_{m} g$, in contradiction with (3). So we conclude that $x \in C+0^{+} F$, which proves the required inclusion $\mathrm{F \subset CC}+0^{+} \mathrm{F}$ and hence the equality of these two sets.

Equivalence (b) $\Longleftrightarrow$ (c) is an immediate consequence of Lemma 24, taking into account that faces of closed sets are closed [12, Corollary 18.1.1] and, therefore, their recession cones are closed [12, Theorem 8.2] too. 
In view of the proof of $(\mathrm{b}) \Longrightarrow(\mathrm{a})$, if a compact set $C \subset \mathbb{R}^{n}$ satisfies the property stated in (i) then $F=C+0^{+} F$; hence, by Proposition 3, conv $C$ is a compact component of $F$. Conversely, if conv $C$ is a compact component of $F$ then, by the proof of (a) $\Longrightarrow$ (b), for every $a$ as in (i), we have $G^{*}(a) \cap \operatorname{conv} C \neq \emptyset$ and therefore, since $G^{*}(a)$ is a face of $F$, we also have $G^{*}(a) \cap C \neq \emptyset$.

The following example shows that neither condition (i) nor condition (ii) in Theorem 25 is superfluous.

Example 26. The non $M$-decomposable set $F$ of Example 16 satisfies condition (i) of Theorem 25, with $C:=[0,1] \times[0,1]$, but not condition (ii) This shows that condition (ii) is not superfluous in the statement of Theorem 25. On the other hand, every closed convex set $F$ satisfies (ii) even if it is not $M$-decomposable; hence condition (i) is not superfluous either.

Remark 27. >From Theorem 25 we obtain again Corollary 20. Indeed, combine Corollary 9 with equivalence (a) $\Longleftrightarrow(c)$ in Theorem 25, taking into account that $F$ is a face of itself [12, $p$. 162] and all of its faces are closed.

The following proposition will be useful for proving another property of Mpredecomposable sets mentioned in Section 1, namely that the result on the existence of a minimal decomposition of an M-decomposable set [4, Theorem 19] holds also for M-predecomposable sets.

Proposition 28. If $C$ is a compact component of the M-predecomposable set $F$, then $\operatorname{ext} F \subset C$.

Proof. If $z \in \operatorname{ext} F \backslash C$, then $z=c+t d$ with $c \in C, 0 \neq d \in 0^{+} F$ and $t>0$. Note that $z$ is the midpoint of the segment with extremes in $c$ and $c+2 d$, both of which belong to $F$, in which case $z \notin e x t F$, so that we get a contradiction.

We prove now the announced result on the existence of minimal decompositions of M-predecomposable sets.

Theorem 29. If $F \subset \mathbb{R}^{n}$ is $M$-predecomposable and $c l 0^{+} F$ is pointed, then there exists a smallest compact component of $F$, i.e., there exists a compact convex set $C^{\prime}$ such that $F=C^{\prime}+0^{+} F$ and for every compact convex set $C$ such that $F=C+0^{+} F$, it holds that $C^{\prime} \subset C$. Moreover $C^{\prime}=c l$ conv ext $F$.

Proof. Let $C$ be a compact component of $F$. Let $C^{\prime}:=c l$ conv ext $F$, and $\widehat{C}:=c l$ conv ext $c l F$. We claim that $\widehat{C} \subset C^{\prime}$. Take $z \in \operatorname{ext} c l F$. By Proposition 28 , we have $z \in C \subset F$. Since every extreme point of a convex set is also an extreme point of every convex subset it belongs to [12, p. 163], we conclude that $z \in \operatorname{ext} F$. We have proved that ext $c l F \subset \operatorname{ext} F$, so that

$$
\widehat{C} \subset C^{\prime},
$$

establishing the claim.

Note that $\widehat{C} \neq \emptyset$ as a consequence of [4, Proposition 19]. It follows from (4) that $C^{\prime} \neq \emptyset$. In view of Proposition 28, we have $C^{\prime} \subset \widetilde{C}$ for every compact 
convex set $\widetilde{C}$ such that $F=\widetilde{C}+0^{+} F$, so that in order to establish the result, it suffices to prove that

$$
F=C^{\prime}+0^{+} F \text {. }
$$

We get from Proposition 8 and [4, Proposition 19] that $c l F=\widehat{C}+c l 0^{+} F$. Consider now the convex set $\widehat{F}:=\widehat{C}+0^{+} F$. Note that $c l F=C+c l 0^{+} F$, and $c l \widehat{F}=\widehat{C}+c l 0^{+} F=c l F$. So $F$ and $\widehat{F}$ have the same closure. It follows that both convex sets have also the same relative interior [12, Theorem 6.3].

Now we proceed to prove that (5) holds. Notice first that, since $C^{\prime} \subset C$ and $C+0^{+} F=F$, we have $C^{\prime}+0^{+} F \subset F$. In order to prove the opposite inclusion, in view of [12, Theorem 18.2] we only need to prove that the relative interior of every face $Q$ of $F$ is contained in $C^{\prime}+O^{+} F$. This inclusion certainly holds for $Q:=F$, since in this case $r i F=r i \widehat{F}=r i\left(\widehat{C}+0^{+} F\right) \subset \widehat{C}+0^{+} F \subset C^{\prime}+0^{+} F$. For an arbitrary face $Q$, by applying this observation to the M-predecomposable set $Q$ (see Proposition 14), we conclude that $r i Q \subset \operatorname{cl} \operatorname{conv} \operatorname{ext} Q+O^{+} Q \subset$ $C^{\prime}+O^{+} F$, the latter inclusion following from ext $Q \subset \operatorname{ext} F$ (see [12, p. 163]) and $O^{+} Q \subset O^{+} F$ (see Theorem 15). Thus, (5) holds, completing the proof.

We mention that the discussion of minimal decompositions of M-decomposable sets whose recession cones are not pointed, presented in Section 1, holds "verbatim" for M-predecomposable sets. Given an M-predecomposable set $F=$ $C+D$, we can write it as $F=L+\left(C+D^{\prime}\right)$, where $L$ is the linearity of $F$ and $D^{\prime \perp}$. $D^{\prime}$ is pointed, and hence, in view of Theorem $29, C+D^{\prime}$ admits a minimal decomposition $\left(\bar{C}, D^{\prime}\right)$. If we look now at the decomposition $F=$ $\bar{C}+\left(L+D^{\prime}\right)=\bar{C}+D$, it is indeed minimal in the weak sense, i.e. there exists no compact and convex set $C^{\prime}$ strictly contained in $\bar{C}$ such that $F=C^{\prime}+D$, but $\bar{C}$ is not unique, and it could be replaced in the decomposition of $F$ by $a+\bar{C}$ for any $a \in L$. The uniqueness is recovered if we adopt the convention that the compact component $C$ in the decomposition of an M-predecomposable set $F$ must be contained in $L^{\perp}$, where $L$ is the linearity of $F$, which can be seen also as the largest linear subspace contained in the recession cone of $F$. Note that such convention is vacuous when the recession cone of $F$ is pointed, because in such case $L=\{0\}$ and hence $L^{\perp}=\mathbb{R}^{n}$.

\section{References}

[1] Cottle, R.W., Pang, J.S., Stone, R.S. The Linear Complementarity Problem. Academic Press, New York (1992).

[2] Daniilidis, A., Martínez-Legaz, J.E. Characterization of evenly convex sets and evenly quasiconvex functions. Journal of Mathematical Analysis and Applications 273 (2002) 58-66.

[3] Dantzig, G.B. Linear Programming and Extensions. Princeton University Press, Princeton (1963). 
[4] Goberna, M.A., González, E., Martínez-Legaz, J.E., Todorov, M.I. Motzkin decomposition of closed convex sets. Journal of Mathematical Analysis and Applications 364 (2010) 209-221.

[5] Goberna, M.A., Iusem. A.N., Martínez-Legaz, J.E., Todorov, M.I. Motzkin decomposition of closed convex sets via truncation. Journal of Mathematical Analysis and Applications 400 (2013) 35-47.

[6] Goberna, M.A., Martínez-Legaz, J.E., Todorov, M.I. On Motzkin decomposable sets and functions. Journal of Mathematical Analysis and Applications 372 (2010) 525-537.

[7] Martínez-Legaz, J.E. Exact quasiconvex conjugation. Zeitschrift für Operations Research, Serie A $\mathbf{2 7}$ (1983) 257-266.

[8] Martínez-Legaz, J.E. Lexicographical characterization of the faces of convex sets. Acta Mathematica Vietnamita 22 (1997) 207-211.

[9] Martínez-Legaz, J.E. Singer, I., Lexicographical separation in $\mathbb{R}^{n}$. Linear Algebra and Its Applications 90 (1987) 147-163.

[10] Motzkin, T. Beiträge zur Theorie der linearen Ungleichungen. Inaugural Dissertation 73 S., Basel (1936).

[11] Pallaschke, D. Urbański, R. On the separation and order law of cancellation for bounded sets. Optimization 51 (2002) 487-496.

[12] Rockafellar, R.T. Convex analysis. Princeton University Press, Princeton (1970). 